\title{
Distribuição Espacial de Lutzomyia longipalpis (Lutz \& Neiva, 1912) e Lutzomyia cruzi (Mangabeira, 1938) no Estado de Mato Grosso
}

\author{
Spatial Distribution of Lutzomyia longipalpis (Lutz \& Neiva, 1912) \\ and Lutzomyia cruzi (Mangabeira, 1938) in the State of Mato Grosso
}

\author{
Nanci Akemi Missawa ${ }^{1,2}$ e Giovana Belem Moreira Lima ${ }^{1}$
}

\begin{abstract}
RESUMO
A leishmaniose visceral é considerada atualmente uma doença emergente e reemergente, em zonas rurais e urbanas, tanto em área domiciliar quanto peridomiciliar. Este trabalho teve como objetivo verificar a distribuição espacial de Lutzomyia longipalpis e Lutzomyia cruzi no Estado de Mato Grosso. Os dados de 1996 a 2004 foram obtidos junto ao Laboratório de Entomologia, cujas capturas foram realizadas com armadilha de luz CDC. Foram pesquisados 68 dos 139 municípios do estado. Lutzomyia longipalpis e Lutzomyia cruzi ocorreram em 23 e 22 municípios, respectivamente. Os resultados demonstraram a grande ocorrência de Lutzomyia longipalpis nas áreas com bioma de floresta, de transição e de cerrado. Lutzomyia cruzi ocorreu principalmente em municípios com área de pantanal e cerrado. A verificação da distribuição da população de vetores no estado e os biomas preferenciais proporcionam indicar áreas vulneráveis e/ou receptivas para a transmissão da doença.
\end{abstract}

Palavras-chaves: Lutzomyia longipalpis. Lutzomyia cruzi. Leishmaniose visceral. Mato Grosso.

\begin{abstract}
Visceral leishmaniasis is currently considered an emergent and reemergent disease, in agricultural and urban zones, both in domestic and peridomestic areas. The objective of this work was to verify the spatial distribution of Lutzomyia longipalpis and Lutzomyia cruzi in Mato Grosso State. Data from 1996 to 2004 was collected by the Entomology Laboratory, whose captures were carried with a CDC light trap. Sixty-eight of the 139 cities in the state have been studied. Lutzomyia longipalpis and Lutzomyia cruzi occurred in 23 and 22 cities, respectively. The results demonstrate the extensive occurrence of Lutzomyia longipalpis in areas with different vegetation types: savanna, transition and Amazon forest. Lutzomyia cruzi occurred mainly in cities with areas of marshland and savanna. Verification of the distribution of the vector populations in the state and their preferential vegetation type, provides an indication of vulnerable and/or receptive areas for disease transmission.
\end{abstract}

Key-words: Lutzomyia longipalpis. Lutzomyia cruzi. Visceral leishmaniasis. Mato Grosso.

Os impactos ambientais no Estado de Mato Grosso, causados pela ocupação desordenada associada às precárias condições de vida das populações humanas, proporcionaram a domiciliação de espécies silvestres, dentre elas as causadoras de doenças ao homem, como as vetoras das leishmanioses ${ }^{2}$.

A leishmaniose visceral tem ampla distribuição ocorrendo na Ásia, na Europa, no Oriente Médio, na África e nas Américas, onde também é denominada leishmaniose visceral americana (LVA) ou calazar neotropical. Atualmente, é considerada como uma doença emergente e reemergente, tanto em zonas rurais como urbanas ${ }^{17}$ e ocorre em área domiciliar ou peridomiciliar ${ }^{14}$.

As transformações no ambiente, decorrentes da migração do homem da área rural para a periferia das cidades, aumentam o número de moradias irregulares, onde há carência de saneamento básico e, por conseguinte, muitas desigualdades sociais. Tais modificações ambientais favorecem ainda, a sobrevivência dos agentes causadores

\footnotetext{
1. Laboratório de Entomologia da Gerência de Vigilância de Vetores e Antropozoonoses da Coordenadoria de Vigilância em Saúde Ambiental da Superintendência de Vigilância em Saúde da Secretaria Estadual de Saúde de Mato Grosso, Cuiabá, MT. 2. Centro de Pesquisas René Rachou da Fundação Oswaldo Cruz, Belo Horizonte, MG. Endereço para correspondência: Dra. Nanci Akemi Missawa. Laboratório de Entomologia. Av. Adauto Botelho s/nº Parque da Saúde, Bairro Coxipó da Ponte, 78085-200 Cuiabá, MT, Brasil.

Telefax: 5565 3661-2494

e-mail: nanci@cpqrr.fiocruz.br

Recebido para publicação em 19/12/2005

Aceito em 3/6/2006
} 
dessas doenças, onde o cão é o mantenedor da cadeia epidemiológica, podendo ou não desenvolver o quadro clínico da doença.

0 principal vetor é o flebotomíneo L. longipalpis ${ }^{6}{ }^{16}$ e 0 L. cruzi é o vetor da Leishmania chagasi na área de Corumbá e Ladário no estado de Mato Grosso do Sul ${ }^{22}$.

Lutzomyia longipalpis ocorre nas regiões norte, nordeste, centro oeste e sudeste e $L$. cruzi ocorre nas mesmas regiões com exceção da região norte ${ }^{4}$. Com o registro da ocorrência de transmissão autóctone de leishmaniose visceral em Mato Grosso $0^{13}$ e a ocorrência de focos no estado ${ }^{514}$, a vigilância entomológica passa a ter atenção e cuidados especiais às áreas em que se observa a ocorrência do vetor e da doença.

No Estado de Mato Grosso, foram realizados estudos que abordaram a ocorrência de $L$. longipalpis ${ }^{7}$ e a descrição da distribuição espacial de flebotomíneos, com ênfase às espécies relacionadas à transmissão das leishmanioses ${ }^{19}$.

A presença do L. longipalpis foi constatada na região metropolitana de Várzea Grande ${ }^{1821}$. A mesma espécie e 0 L. cruzi foram observadas na área de Aproveitamento Múltiplo de Manso (APM-Manso) ${ }^{20}$. Em Mato Grosso, foram realizados estudos relacionados à domiciliação e à antropofilia de L. longipalpis e $L$. cruzr $P^{8}$.

Segundo dados da Secretaria de Vigilância em Saúde do Ministério da Saúde, divulgados em outubro de 2003, na Reunião Nacional de Leishmanioses, o Estado de Mato Grosso possui município de transmissão intensa de leishmaniose visceral (Várzea Grande) e de transmissão moderada (Barra do Garças) além de outros municípios de transmissão esporádica e áreas vulneráveis e/ou receptivas. O Programa de Controle da Leishmaniose Visceral (PCLV) propõe investigações entomológicas para que sejam delineadas as ações de controle da doença $\mathrm{a}^{12}$.

Estudos sobre a biologia de flebotomíneos e sua relação com a região geográfica e o bioma onde ocorrem, contribuem para uma melhor compreensão da dinâmica de transmissão. Este trabalho teve como objetivo verificar a distribuição dos vetores da leishmaniose visceral, $L$. longipalpis e $L$. cruzi, no Estado de Mato Grosso, identificando assim as áreas de transmissão ou de risco, propensas à vigilância entomo-epidemiológica.

\section{MATERIAL E MÉTODOS}

Área de estudo. 0 Estado de Mato Grosso acolhe em seu território três dos mais importantes biomas brasileiros, a floresta amazônica, o cerrado e o pantanal, além de uma vasta área de transição entre floresta e cerrado. No estado, estão as nascentes das Bacias Amazônica e Platina, duas das maiores bacias hidrográficas do continente. Apresenta, ainda, uma ampla diversidade de paisagens e de espécies de fauna e flora. A distribuição das chuvas no decorrer do ano evidencia o caráter tropical da área, com duas estações bem definidas, uma seca e outra chuvosa ${ }^{10}$.

Coleta de dados. Os dados utilizados na pesquisa foram obtidos a partir de relatórios de pesquisas entomológicas de flebotomíneos, realizadas pelo Núcleo de Entomologia (Fundação Nacional de Saúde), atualmente Laboratório de Entomologia (Secretaria de Estado da Saúde) em vários municípios do Estado de Mato Grosso, entre os anos de 1996 e 2004.

As coletas foram realizadas com armadilhas de luz do tipo CDC, instaladas no entardecer e recolhidas cerca da 12 horas depois, ao amanhecer. Os insetos foram capturados tanto dentro das residências (intradomiciliar) quanto nos anexos e/ou abrigos de animais domésticos (peridomiciliar).

Após triagem, os espécimens foram clarificados, montados e identificados ${ }^{23}$. Exemplares estão depositados no Laboratório de Entomologia da Secretaria Estadual de Saúde de Mato Grosso.

\section{RESULTADOS}

Entre os anos de 1996 e 2004, foi realizada pesquisa entomológica em 68 dos 139 municípios do Estado de Mato Grosso, correspondendo a $48,9 \%$. Os vetores da leishmaniose visceral ocorreram em 31 (45,6\%) dos 68 municípios pesquisados. Dentre os 31 municípios, dois apresentaram bioma exclusivamente de floresta, um de transição, sete de cerrado e um de pantanal. Como pode ocorrer diferentes biomas, simultaneamente, foram observados dois municípios com floresta e cerrado, cinco com transição e cerrado, sete com floresta, transição e cerrado, quatro com cerrado e pantanal e dois com floresta e transição (Figura 1).

0 principal vetor para a transmissão da leishmaniose visceral, Lutzomyia longipalpis, foi capturado em 23 (74,2\%) municípios sendo eles Água Boa, Alto Araguaia, Barra do Garças, Cáceres, Canarana, Chapada dos Guimarães, Comodoro, Diamantino, Feliz Natal, Gaúcha do Norte, Juína, Marcelândia, Mirassol d'Oeste, Nobres, Nossa Senhora do Livramento, Nova Brasilândia, Paranatinga, Poconé, Pontal do Araguaia, Rondonópolis, Rosário Oeste, Sorriso e Várzea Grande.

Considerando-se o bioma pesquisado, L. longipalpis ocorreu em $14(93,3 \%)$ dos municípios que apresentaram bioma com floresta amazônica; 12 (75\%) com transição; $18(75 \%)$ com cerrado e 2 (40\%) com pantanal.

Lutzomyia cruzi ocorreu em 22 (71\%) municípios, Alto Araguaia, Barão de Melgaço, Barra do Garças, Canarana, Chapada dos Guimarães, Dom Aquino, Feliz Natal, Itiquira, Jaciara, Juína, Mirassol d'Oeste, Nobres, Nova Brasilândia, Nova Canaã do Norte, Paranatinga, Poconé, Pontal do Araguaia, Poxoréo, Primavera do Leste, Rosário Oeste, Santo Antônio do Leverger e Sorriso.

A espécie $L$. cruzi ocorreu em 9 (60\%) dos municípios com floresta amazônica; $11(68,8 \%)$ com transição; $17(70,8 \%)$ com cerrado e 4 (80\%) com pantanal.

\section{DISCUSSÃo}

Os resultados demonstraram a grande ocorrência de L. longipalpis nas áreas com bioma de floresta, transição e de cerrado. Em trabalhos realizados no Estado de Mato Grosso, foi observada a ocorrência dos maiores índices armadilha, ou seja, das maiores densidades da espécie em região de cerrado ${ }^{19}$. 


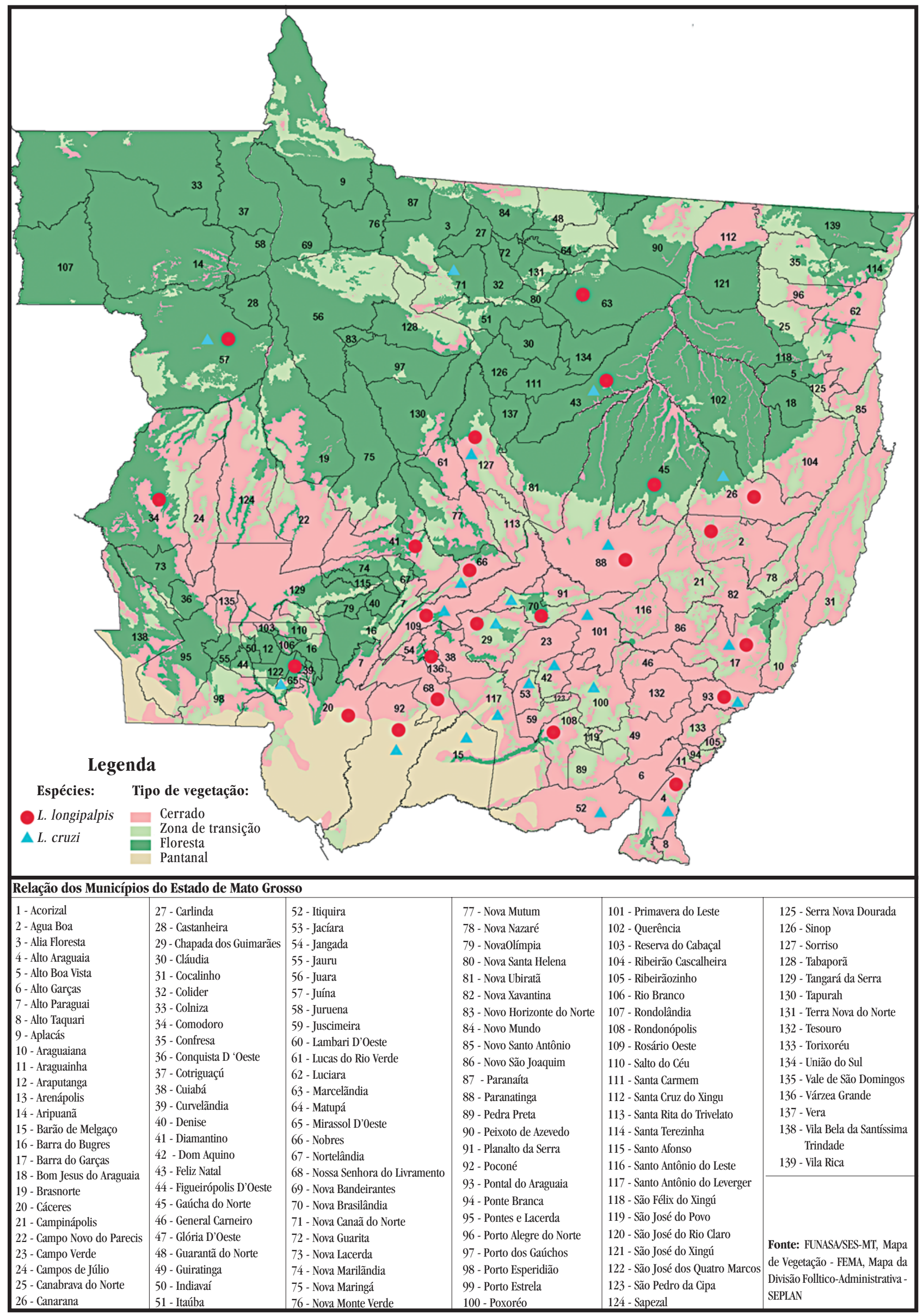

Figura 1 - Distribuição espacial de Lutzomyia longipalpis e Lutzomyia cruzi no Estado de Mato Grosso, Brasil. 
Foram capturados exemplares na maioria dos municípios pesquisados com bioma florestal, sugerindo ser esse um criadouro em potencial ${ }^{15}$, apesar de que $L$. longipalpis não foi observado em pesquisa realizada em Alta Floresta, região sul da Amazônia ${ }^{9}$ e em Peixoto de Azevedo, norte do Estado de Mato Grosso ${ }^{3}$.

As maiores frequiências de $L$. cruzi foram observadas em municípios com área de pantanal e cerrado, de acordo com outras pesquisas realizadas, indicando ser o cerrado, o ambiente preferencial da espécie ${ }^{19}$.

A ocorrência de $L$. longipalpise $L$. cruzi nos diferentes biomas do estado com ampla distribuição, confirma o caráter generalizado das espécies, adaptadas a habitats diversificados ${ }^{1}$. A manutenção de $L$. longipalpis nos ecótopos estudados provavelmente deve-se à sua preferência alimentar eclética, possuindo o vetor, capacidade de picar o homem, o cão, as aves e outros animais ${ }^{4}$.

A verificação da dispersão da população de vetores no estado e os biomas preferenciais proporcionam indicar áreas vulneráveis e/ou receptivas para a transmissão da leishmaniose visceral, dados que podem ser utilizados para subsidiar ações de prevenção e controle da doença.

A vigilância entomo-epidemiológica deve adotar medidas preventivas que permitam acompanhar seguidamente 0 comportamento do vetor e o estado de saúde populacional. Tratase, pois de um conjunto de atividades em Saúde Pública, destinado a englobar as informações necessárias para o conhecimento, em qualquer tempo, da situação do quadro epidemiológico em foco, sendo possível detectar, ou mesmo prever, as alterações, direcionando e orientando a aplicação de medidas preventivas, com o propósito de atingir o máximo de eficácia.

0 trabalho integrado entre as Secretarias Municipais de Saúde e a Secretaria de Estado de Saúde se faz de extrema importância, pois permite otimizar os recursos e a efetividade das ações de controle do vetor, diminuindo assim o risco de transmissão da doença, garantindo a melhoria da qualidade de vida da população.

\section{AGRADECIMENTOS}

Nossos agradecimentos aos técnicos do Laboratório de Entomologia da Secretaria Estadual de Saúde de Mato Grosso pela contribuição nas atividades de campo e de laboratório, imprescindíveis para o desenvolvimento desse trabalho.

\section{REFERÊNCIAS BIBLIOGRÁFICAS}

1. Aguiar GM, Medeiros, WM. Distribuição e habitats. In: Rangel EF, Lainson R (orgs) Flebotomíneos do Brasil. Editora da Fundação Oswaldo Cruz, Rio de Janeiro, p.207-255, 2003.

2. Araújo JC, Rebêlo JMM, Carvalho ML, Barros VLL. Composição dos flebotomíneos (Diptera, Psychodidae) do município de Raposa-MA, Brasil. Área endêmica de Leishmaniose. Entomología y Vectores 7 (suppl 1): 33-47, 2000.

3. Azevedo ACR, Souza NA, Meneses CRV, Costa WA, Costa SM, Lima JB, Rangel, EF. Ecology of sand flies (Diptera: Psychodidae: Phlebotominae) in the north of state of Mato Grosso, Brazil. Memórias do Instituto Oswaldo Cruz 97 (supl 4): 459-464, 2002.
4. Brazil RP, Gomes Brazil B. Bionomia. In: Rangel EF, Lainson R (orgs) Flebotomíneos do Brasil. Editora da Fundação Oswaldo Cruz, Rio de Janeiro, p.257-274, 2003.

5. Camiá RP, Rinaldi J, Fontes CJF, Hueb M. Foco de Leishmaniose Visceral em Mato Grosso. XV Reunião Anual de Pesquisa Aplicada em Doença de Chagas e III Reunião de Pesquisa Aplicada em Leishmanioses. Revista da Sociedade Brasileira de Medicina Tropical 32 (supl 2):127, 1999.

6 Carrera M. Insetos de Interesse Médico e Veterinário. Editora Universidade Federal do Paraná, Curitiba, 1991.

7. CIPA Group: Bermudes H, Dedet JP, Falcão AL, Feliciangeli D, Ferro C, Galati EAB, Gomes EL, Herrero MV, Hervas D, Lebbe J, Morales A, Oguzuku E, Rangel EF, Sherlocki IA, Torres M. Vignes R, Wolf M. Data base. Computer aided identification of phlebotomine sandflies of America, http://cipa.snv/jussieu.fr, 1999.

8. Costa LB, Bertúlio MA, Rodrigues H, Maciel GB, Filho VC, Ramos SR, Souza CO, Silva AM, Barros IM. Ocorrência de Flebotomíneos Vetores da Leishmaniose Visceral nos Municípios do Estado de Mato Grosso. Congresso de Saúde Coletiva, Brasília, Distrito Federal. Revista Abrasco 8 (supl 2): 639, 2003.

9. De Luca AS, Vasconcelos HL, Barrett TV. Distribution of sandflies (Diptera: Phlebotominae) in forest remnants and adjacent matrix habitats in Brazilian Amazonia. Brazilian Journal Biology 63 (suppl 3): 401-410, 2003.

10. Ferreira JCV. Mato Grosso e seus Municípios. Editora Buriti, Cuiabá-MT, 2001.

11. Fundação Nacional da Saúde. Doenças Infecciosas e Parasitárias: aspectos clínicos, de vigilância epidemiológica e de controle - Guia de Bolso. Ministério da Saúde, Brasília, 1998.

12. Fundação Nacional de Saúde. Manual de Vigilância e Controle da Leishmaniose Visceral. Brasil, Ministério da Saúde, Brasília, 2003.

13. Hueb M, Assis SB, Guimarães EED, Rosa DL, Fontes CJF. Ocorrência de Transmissão Autóctone de Leishmaniose Visceral em Mato Grosso. Revista da Sociedade Brasileira de Medicina Tropical 29 (supl 3): 281-282, 1996.

14. Hueb M, Camiá RP, Ribeiro LC, Fontes CJF. Calazar em Mato Grosso - Foco Recente em Área Periurbana. Revista da Sociedade Brasileira de Medicina Tropical 33 (supl 1): 324-325, 2000.

15. Lainson R, Rangel EF. Ecologia das Leishmanioses. In: Rangel EF, Lainson R (orgs) Flebotomíneos do Brasil. Editora da Fundação Oswaldo Cruz, Rio de Janeiro, p.291-309, 2003.

16. Marcondes CB. Entomologia Médica e Veterinária. Editora Atheneu, São Paulo, 2001.

17. Neves DP. Parasitologia Humana. Editora Atheneu, São Paulo, 2000.

18. Ribeiro ALM, Missawa NA. Ocorrência de Flebotomíneos no Município de Várzea Grande/ Mato Grosso, no período de abril a setembro de 2001. In: Simpósio Amazônia e Ambiente, 2002.

19. Ribeiro ALM, Missawa NA. Spatial distribution of phlebotomine species in the state of Mato Grosso, Brazil, in the period of 1996 to 2001. Entomología y Vectores 9: 33-34, 2002.

20. Ribeiro ALM, Oliveira RC, Miyazaki RD, Pignat WA. Inventário dos vetores da Leishmaniose (Diptera: Psychodidae: Phlebotominae) em Área de Aproveitamento Múltiplo de Manso, Chapada dos Guimarães, Nobres, Rosário Oeste e Nova Brasilândia/Mato Grosso/Brasil (Dados preliminares). Revista da Sociedade Brasileira de Medicina Tropical 35 (supl 1): 313, 2002.

21. Ribeiro ALM, Rodrigues JSV, Biancardi CMAB. Ocorrência de Lutzomyia longipalpis (Diptera - Psychodidae) na Região Metropolitana de Várzea Grande - Mato Grosso. In: Resumos do XXIII Congresso Brasileiro de Zoologia, p. 327, 2000.

22. Santos SO, Arias J, Ribeiro AA, Hoffmann MP, Freitas RU, Malacco MAF. Incrimination of Lutzomyia cruzi as a vector of American Visceral Leishmaniasis. Medical and Veterinary Entomology 12:315-317, 1998.

23. Young DG, Duncan MA. Guide to the identification and geografic distribuition of Lutzomyia sand files in México, the West Indies, Central and South America (Diptera: Psychodidae). Associated Publishers American Entomological Institute. Florida, 1994. 